\title{
Dependence of Plasmonic Properties of Silver Island Films on Nanoparticle Size and Substrate Coverage
}

\author{
M. G. Sreenivasan, ${ }^{1,2}$ Shweta Malik, ${ }^{1}$ Srikanth Thigulla, ${ }^{2}$ and Bodh Raj Mehta ${ }^{1}$ \\ ${ }^{1}$ Thin Film Laboratory, Department of Physics, Indian Institute of Technology Delhi, Hauz Khas, New Delhi 110016, India \\ ${ }^{2}$ Hind High Vacuum Company Private Limited, No. 17, Phase 1, Peenya Industrial Area, Bangalore 560058, India \\ Correspondence should be addressed to M. G. Sreenivasan; mgs@hhv.in
}

Received 10 May 2013; Revised 6 August 2013; Accepted 7 August 2013

Academic Editor: Chunyi Zhi

Copyright (c) 2013 M. G. Sreenivasan et al. This is an open access article distributed under the Creative Commons Attribution License, which permits unrestricted use, distribution, and reproduction in any medium, provided the original work is properly cited.

Localized surface plasmon resonance displayed by metal nanoparticles has been studied in silver island films prepared by the simple technique of vacuum evaporation, which is one of the options that is easily adaptable for large area and low cost applications. Silver island films with varying island sizes and areal coverages are prepared by depositing silver films with varying thicknesses followed by annealing. The optical properties of the samples have been explained in terms of dependence of scattering and absorption on the metal island size, interparticle interaction and matrix effects, and the wavelength range over which the plasmonic effects are present.

\section{Introduction}

Plasmonic properties of metal nanoparticles present novel possibilities of plasmonic interaction with light which can subsequently be used to enhance the performance of solar cells $[1,2]$. Particularly in the case of thin film solar cells, where the light absorption is limited by the thickness of the absorber layer, the use of metal nanoparticles is considered as one of the most promising ways to trap light and thereby achieve increased efficiencies. In line with this, various research groups are working on incorporating metal nanoparticles into solar cell structures and a number of positive results have come out [3-17].

Although a number of methods exist for the synthesis of metal nanoparticles, one of the most commonly used methods for solar cell applications is vacuum evaporation of thin films followed by annealing $[4,5,8]$. This technique results in the formation of nanoparticles in the form of islands due to coalescence when the thin metal film is annealed. This also presents one of the simplest ways for subsequent integration into the production line.

In order to incorporate metal nanoparticles into solar cell structures, it is important to understand the scattering and absorption from the nanoparticles and the wavelength region in which the plasmonic effects are obtained. For a system where the composition of nanoparticle and the substrate is fixed, the plasmonic effects are primarily dependent on the size, shape, and the interparticle distance (areal coverage) of the nanoparticles. In the case of synthesis of nanoparticles by the technique of vacuum evaporation and annealing all these factors vary together as they are dependent upon interrelated deposition parameters. So, even though a fair idea of the optical properties can be obtained from simulations, experimental studies can offer a clearer picture.

In this work, we have studied the effect of film thickness on the particle size and areal coverage of the metal islands obtained upon annealing, and have studied the direct, scattered, and total transmittance and the specular, diffuse, and total reflectance from these films. Based on these studies, we have tried to identify deposition conditions for silver island films that might be suitable for incorporation into solar cell structures.

\section{Experimental}

Deposition of the silver films was done in the presence of argon gas in a custom-built thermal evaporator system. The 
TABLE 1: Table showing the sample labeling, deposition conditions, and the resulting effective diameter and aerial coverage of the metal island films.

\begin{tabular}{|c|c|c|c|c|c|}
\hline \multirow[b]{2}{*}{ Sample no. } & \multicolumn{2}{|c|}{ Deposition condition $^{\mathrm{a}}$} & \multirow[b]{2}{*}{ Effective diameter ${ }^{\mathrm{b}}(\mathrm{nm})$} & \multirow[b]{2}{*}{ Areal coverage $^{c}(\%)$} & \multirow{2}{*}{$\begin{array}{l}\text { Avg. center-to-center } \\
\text { spacing " } s \text { "d }(\mathrm{nm})\end{array}$} \\
\hline & $\begin{array}{l}\text { Thickness of film } \\
(\mathrm{nm})\end{array}$ & Pressure (Torr) & & & \\
\hline 1 & 0.5 & $1 E-3$ & 14 & 13 & 34 \\
\hline 2 & 3.0 & $1 E-3$ & 23 & 31 & 37 \\
\hline 3 & 5.0 & $1 E-3$ & 32 & 35 & 48 \\
\hline 4 & 5.7 & $1 E-4$ & 52 & 38 & 75 \\
\hline 5 & 7.3 & $1 E-4$ & 67 & 41 & 93 \\
\hline 6 & 9.3 & $1 E-4$ & 74 & 43 & 100 \\
\hline 7 & 11.3 & $1 E-4$ & 87 & 39 & 123 \\
\hline 8 & 14.2 & $1 E-4$ & 95 & 39 & 135 \\
\hline
\end{tabular}

${ }^{\text {a }}$ All samples were annealed at $200^{\circ} \mathrm{C}$ for 1 hour at $\sim 5 \times 10^{-6}$ Torr vacuum.

${ }^{b}$ Effective diameter is defined as the diameter of a circle having the same cross-sectional area as that of the metal island [19].

${ }^{c}$ Percentage of surface area covered by silver islands.

dThe average center-to-center spacing " $s$ " is calculated from the values of effective diameter and the aerial coverage.
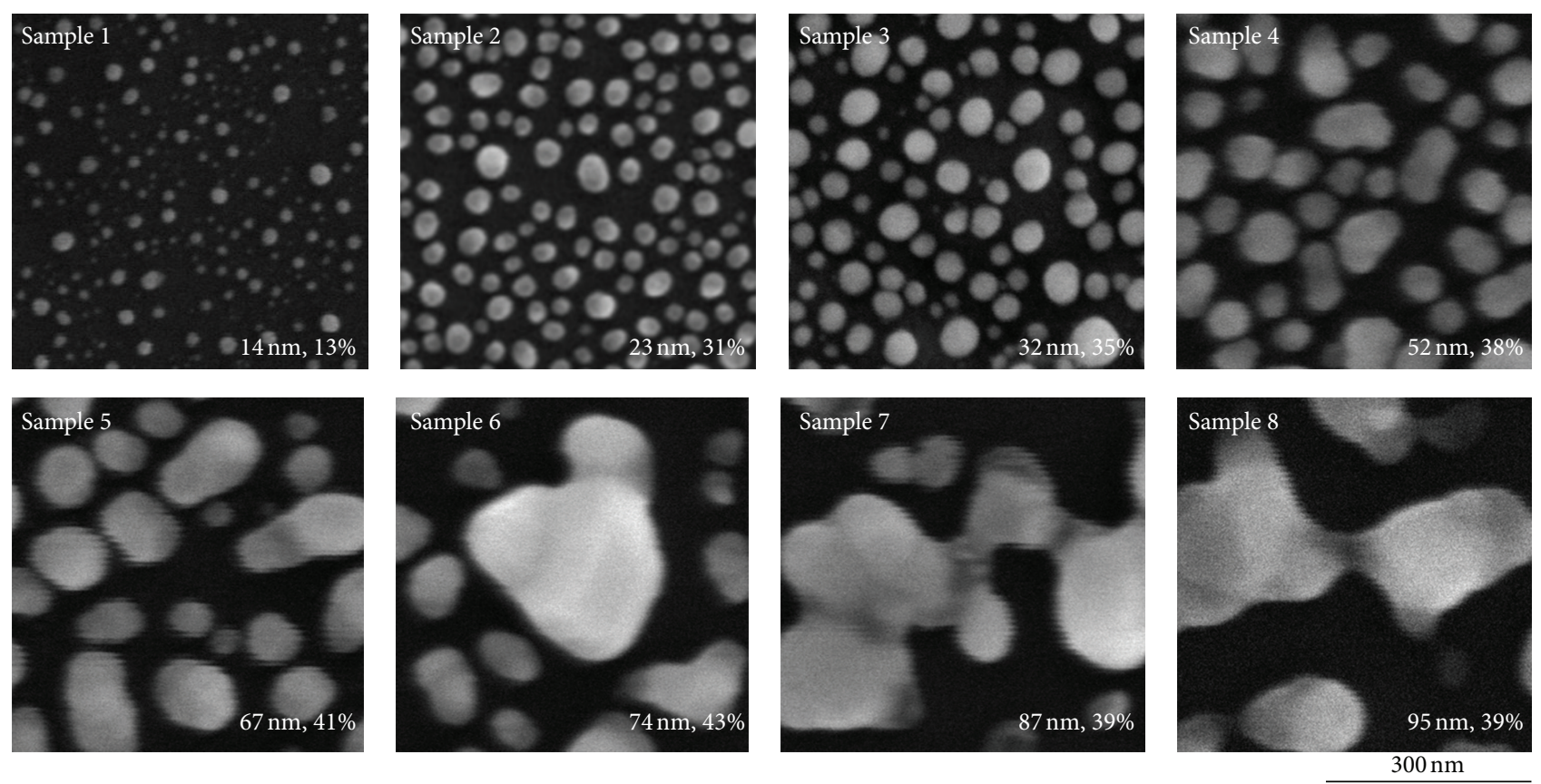

FIGURE 1: SEM images of samples deposited under different deposition conditions on silicon substrates. All the images have the same magnification.

chamber was evacuated to $\sim 5 \times 10^{-6}$ Torr using a combination of rotary and diffusion pumps. Argon gas was then introduced into the chamber using a mass flow controller to obtain the desired pressure. The thicknesses of the films were monitored using a quartz crystal oscillator. Annealing of the samples was carried out within the same thermal evaporator chamber in $\sim 5 \times 10^{-6}$ Torr vacuum. Deposition and annealing were done simultaneously on silicon and glass substrates. The silicon samples were used for scanning electron microscopy (SEM) imaging and the glass substrates were used for optical studies. The SEM imaging was done using a FEI-Quanta-3D system, and the analysis of the images was done using the Image J software [18]. The optical properties of the films were studied using a Perkin Elmer Lambda 35 UV-Visible (UVVis) spectrophotometer and its $50 \mathrm{~mm}$ integrating sphere accessory.

The direct transmittance $\left(T_{\text {dir }}\right)$ was measured using the UV-Vis spectrophotometer in the transmission mode, and the total transmittance $\left(T_{\text {tot }}\right)$ was measured using the integrating sphere accessory by placing the sample at the entrance port. Difference between the two $\left(T_{\text {tot }}-T_{\text {dir }}\right)$ was used to obtain the scattered transmittance $\left(T_{\text {scat }}\right)$. Baseline measurements 


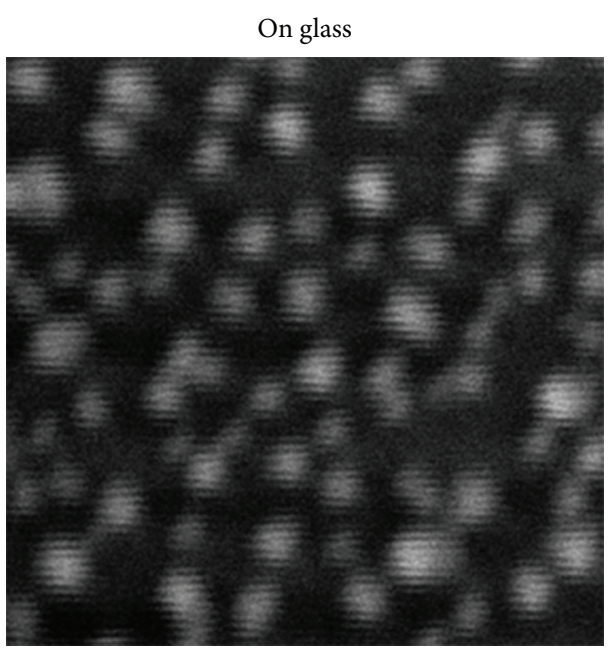

(a)

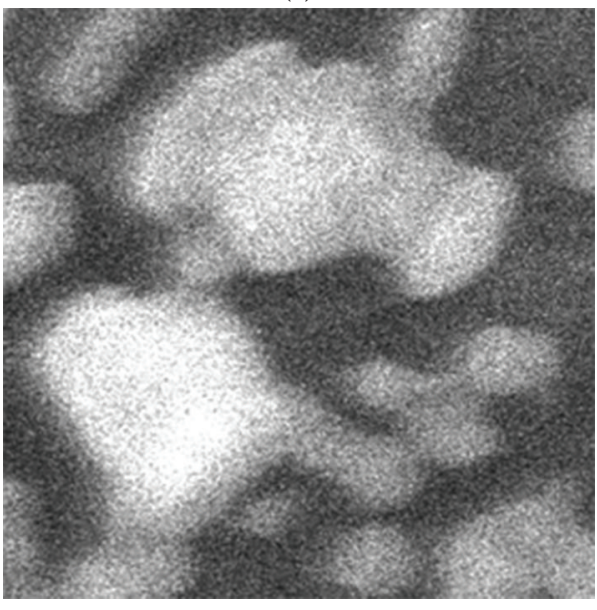

(c)

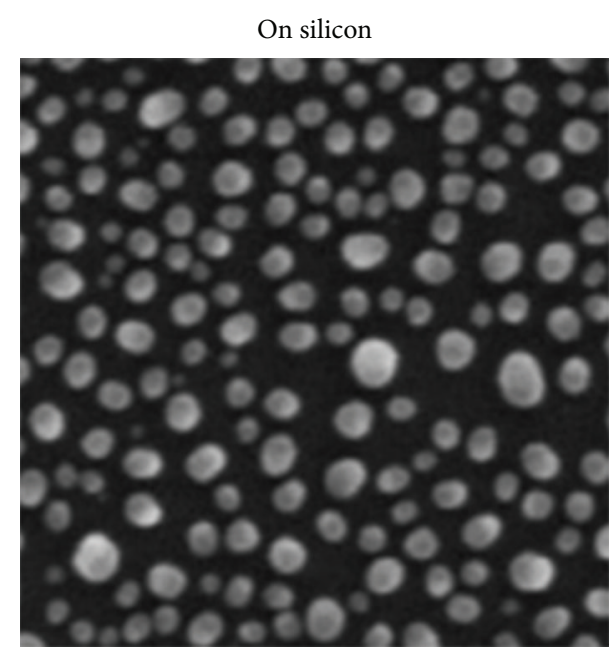

(b)

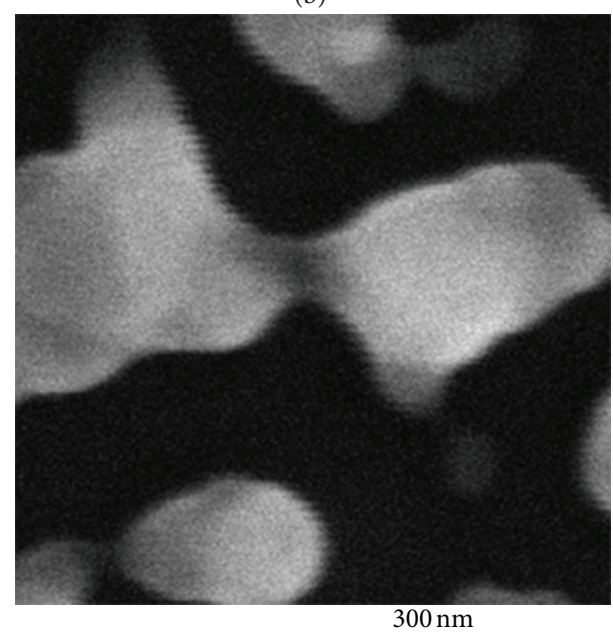

(d)

FIGURE 2: SEM images for comparison of the features obtained on silicon and on glass. (a) and (b) correspond to sample 2, and (c) and (d) correspond to sample 8 .

were done using bare microscope slides to correct for transmission losses in glass. Reflectance measurements were done by placing the sample at the exit port of the integrating sphere accessory in different tilt positions. For measuring the diffuse reflectance $\left(R_{\text {dif }}\right)$, the $0^{\circ}$ holder was used. In this configuration, the light is incident normally onto the sample surface due to which the specular part of the reflected light goes out through the entrance port, and hence only the diffuse part is measured. In order to measure the total reflectance $\left(R_{\text {tot }}\right)$, the $8^{\circ}$ holder accessory (provided by Perkin Elmer) of the integrating sphere is used. By placing the sample on this holder, the incident beam hits the sample surface at an angle of $8^{\circ}$ to the normal. Thus both the specular and diffuse reflections are measured collectively by the detector giving the value of total reflectance. The specular reflectance $\left(R_{\text {spec }}\right)$ is then calculated as a difference between $R_{\text {tot }}$ and $R_{\text {dif }}$.

\section{Results and Discussion}

Silver island films with different sized nanoislands were synthesized by thermal evaporation of silver films of different thicknesses followed by annealing. Table 1 lists the deposition conditions of the silver films and the resultant effective diameter and areal coverage of the silver nanoparticles. The nanoparticle size increases gradually with the increase in film thickness, and so a good control over the size is possible by varying the thickness. But the corresponding areal coverage offers much lesser flexibility, increasing sharply and leveling off at $\sim 40 \%$ for film thicknesses of $\sim 6 \mathrm{~nm}$ and higher. The SEM images of the samples 1-8 are shown in Figure 1. When the as-deposited films have well separated islands, more circular islands are obtained upon annealing as in the case of samples 1-3, whereas when islands start to merge as shown in sample 4 onwards, the shape of islands in the annealed film becomes more irregular and nonspherical.

As the main objective of this work is to study optical properties of nanoislands, the samples were therefore simultaneously deposited onto glass substrate. As the refractive index of the glass substrate is low, the interaction between the nanoparticle and the substrate is minimal, and the optical properties can be considered to be coming mainly from the bare island resonance [19]. In order to make a comparison 


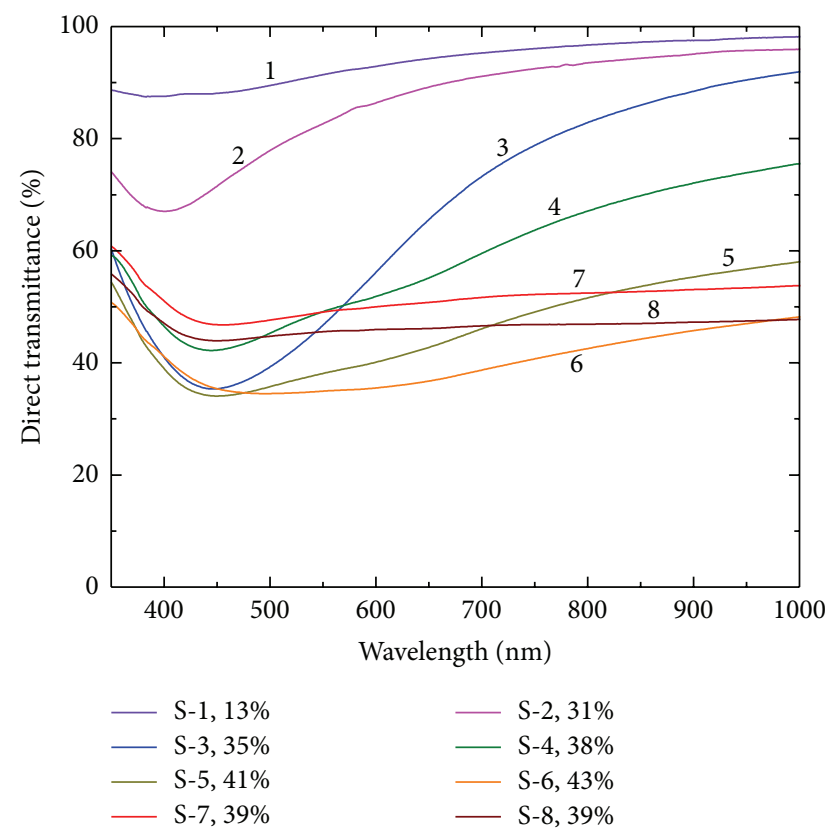

FIGURE 3: Direct transmittance spectra of samples 1-8.

of the nanoparticles formed on glass and silicon substrates, representative samples synthesized under similar conditions were chosen for SEM imaging. Figure 2 shows the SEM images of the silver islands formed on glass and silicon substrates for samples 2 and 8 . The images taken on glass substrates are not well focused due to the effect of charging. The samples deposited on glass substrates tend to be more elongated and have a broader distribution of particles as compared to silicon substrates, but in going from sample 2 to sample 8 , a similar trend in particle size and areal coverage is followed on both glass as well as silicon substrates. Similar observations have been made by other groups as well $[8,19]$.

The optical properties of nanoparticles formed on the glass substrates were characterized using the UV-Vis spectrophotometer and the integrating sphere accessory. In all the measurements, the light was incident onto the silver nanoparticles side of the sample. Figure 3 shows the direct transmittance spectra of samples 1 to 8 . The dip in transmission spectra which is indicative of the localized surface plasmon resonance of the metal nanoparticles is seen for all the samples.

In all the samples, the average center-to-center spacing " $s$ " between the particles is lower than $2.5 d$, where " $d$ " is the effective diameter (see Table 1). The $s / d$ ratio of 2.5 is considered as a threshold below which the field experienced by one particle will be influenced by the field from neighboring particles [20-22]. Hence the inter-particle coupling is expected to have a significant effect on the optical properties of all the samples.

For the case of sample 1, a broad dip is observed which could be due to a combination of dephasing that is observed in particles $<\sim 10 \mathrm{~nm}$ due to size dependant modifications in the dielectric constants due to surface damping [23], combined with inter-particle interaction effects. Sample 2 has a more pronounced dip as compared to sample 1 due to the lesser number of particles with $d<10 \mathrm{~nm}$. The plasmon resonance dip red-shifts and broadens as we move to sample 3. This is due to a combination of increased particle size and increased inter-particle coupling resulting from the decreased inter-particle gaps.

In samples 4 and 5, two peaks can be discerned. The first order peak is further red-shifted and a more prominent second order peak appears. In the case of individual noninteracting nanoparticles in air, particles $\geq \sim 60 \mathrm{~nm}$ are found to exhibit a quadrupole mode, and as the inter-particle spacing is reduced, the second order peak is seen in the case of smaller particles as well [19]. Additionally, from sample 4 onwards, due to island coalescence beginning to take place, the morphology is also changing leading to more close coupling between the particles, thereby leading to more pronounced red-shifting and appearance of higher order peaks from this thickness onwards. In the case of sample 6, due to broadening of the second order peak, there is more overlap between the two peaks. As we move to samples 7 and 8 , we find that the resonance dip becomes much broader and is found to encompass a wide wavelength range. This could have a significant application in the case of improving solar cell performance.

To determine whether the dip in direct transmission is originating from scattering or absorption, the data obtained from integrating sphere measurements were studied. The total transmittance and total reflectance data were used to find the absorptance, and the scattered transmittance and diffuse reflectance spectra were used to find the total scattering. The total transmittance and total reflectance spectra measured for samples 1-8 are shown in Figures 4(a), and 4(b), respectively. The total reflection complements the total transmission spectra. The positions of reflection maxima match the positions of the transmission minima due to the effect of scattering. From these measurements, the absorptance spectra were determined as $100-T_{\text {tot }}-R_{\text {tot }}$ and are plotted in Figure 4(c).

The scattered transmittance and diffuse reflectance spectra for samples 1-8 are shown in Figures 5(a), and 5(b), respectively. The total scattering by the island films, that is, the sum of $T_{\text {scat }}$ and $R_{\text {dif }}$, is plotted in Figure 5(c). As the nanoparticles are formed on a low index (glass) substrate, the scattering is distributed in all directions, whereas, when the nanoparticles are surrounded by a high index medium such as silicon on one side, the scattering will predominantly be directed towards the high index medium [24].

In order to describe the changes in the optical properties as we go from sample 1 to 8 , the total absorptance and total scattering obtained at two wavelengths, namely, $\lambda=400 \mathrm{~nm}$ and $500 \mathrm{~nm}$, have been plotted in Figure 6.

The total transmission follows a similar trend as the direct transmission. The difference between the two, which is the scattered transmission, is shown in Figure 5(a). For samples 1 and 2, the scattered transmission is lower due to the lower scattering efficiency of the smaller particles. In the case of sample 3 , the scattering is lower at resonance because of the dominant absorption cross-section, but as we move to longer wavelengths, we see that the scattering is still present, while 


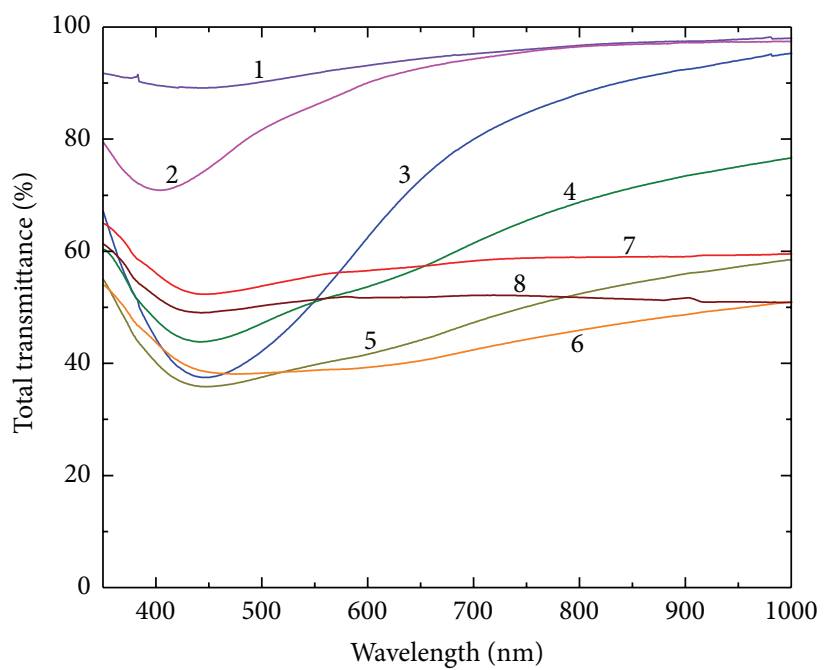

(a)

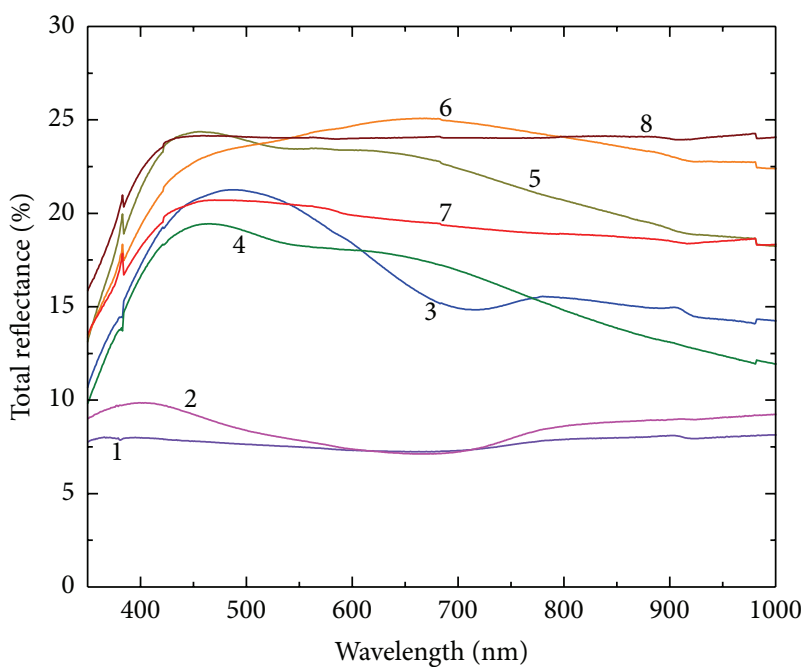

(b)

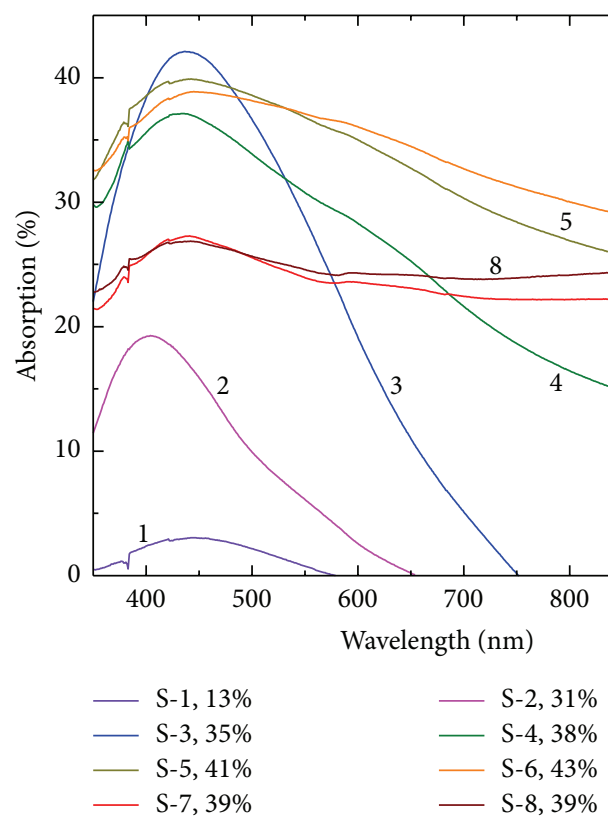

(c)

Figure 4: (a) Total transmittance, and (b) total reflectance measured using integrating sphere; (c) absorptance calculated from (a) and (b).

the absorption comes down. A similar behavior has been theoretically predicted based on finite element calculations by Akimov et al. [25]. For the case of particles of the order of 30$40 \mathrm{~nm}$, at the dipolar resonance wavelength, the absorption cross-section is higher as compared to the scattering crosssection, but as we move away from resonance, the scattering is still present while the absorption comes down significantly. This can be used for enhancing the efficiency of thin film solar cells. Since the lower wavelengths are absorbed and the longer wavelengths are scattered, this nanoparticle size will be ideally suited for the backside of the solar cell wherein only the longer wavelengths reach the nanoparticles.

In the case of samples 4 and 5 , the scattering is decreased as absorption due to the higher order resonance comes into picture due to islands beginning to coalesce. Hence the region just before the islands begin to coalesce may be targeted for applications in solar cells. As the thickness of the as-deposited film is increased further, for samples $6-8$, the scattering is enhanced due to the increased scattering efficiency of the larger nanoparticles and is also present over a wider wavelength range due to the coupling between the particles. Hence this parameter space could also be suitable for solar cell applications.

As we go from sample 1 to 3 , we find that the absorption (Figure 6) goes up steeply. In the range of particle size from $15 \mathrm{~nm}$ to $40 \mathrm{~nm}$, the absorption is expected to increase [25] after which it starts to come down. Additionally, the increased areal coverage could also be contributing to the increased absorption [25]. For samples 4-8, the absorption mainly comes from the higher order mode, and absorption 


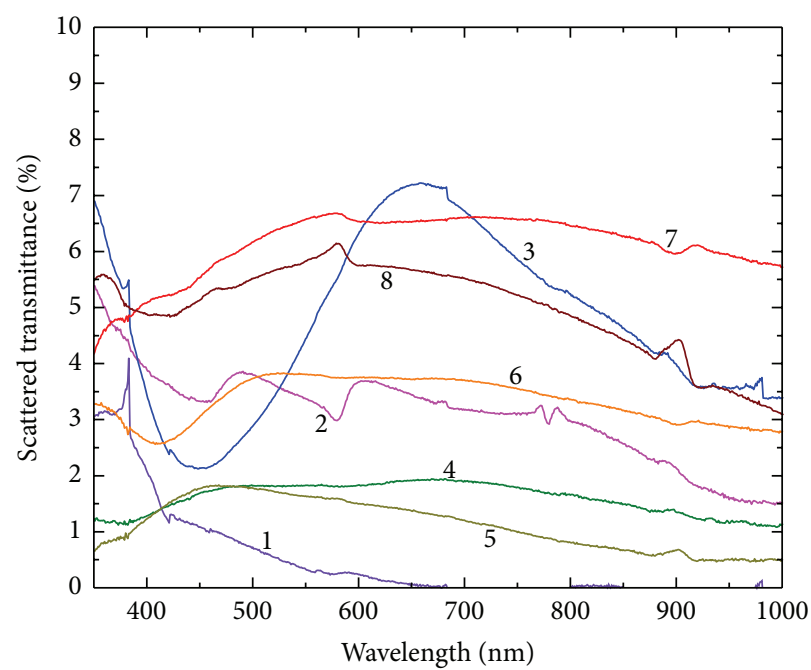

(a)

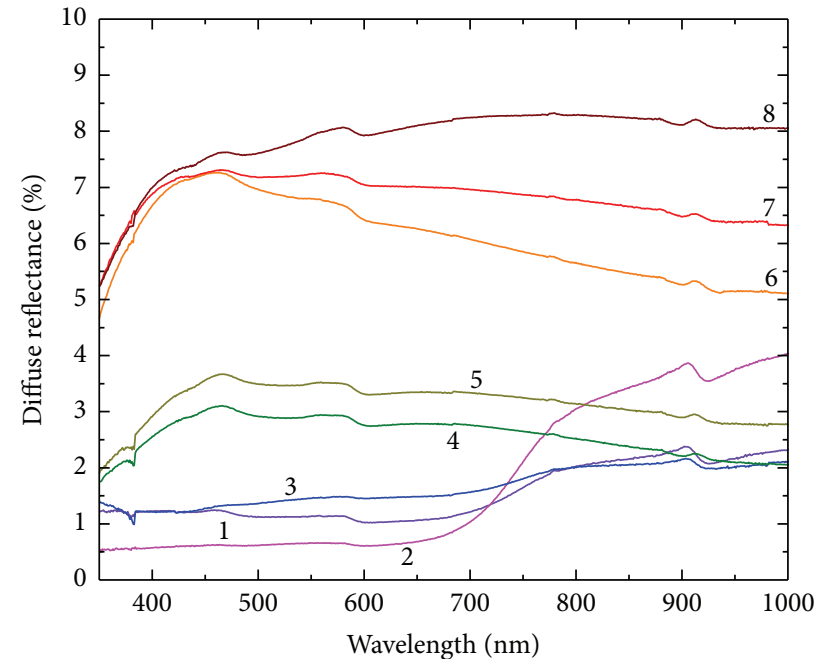

(b)
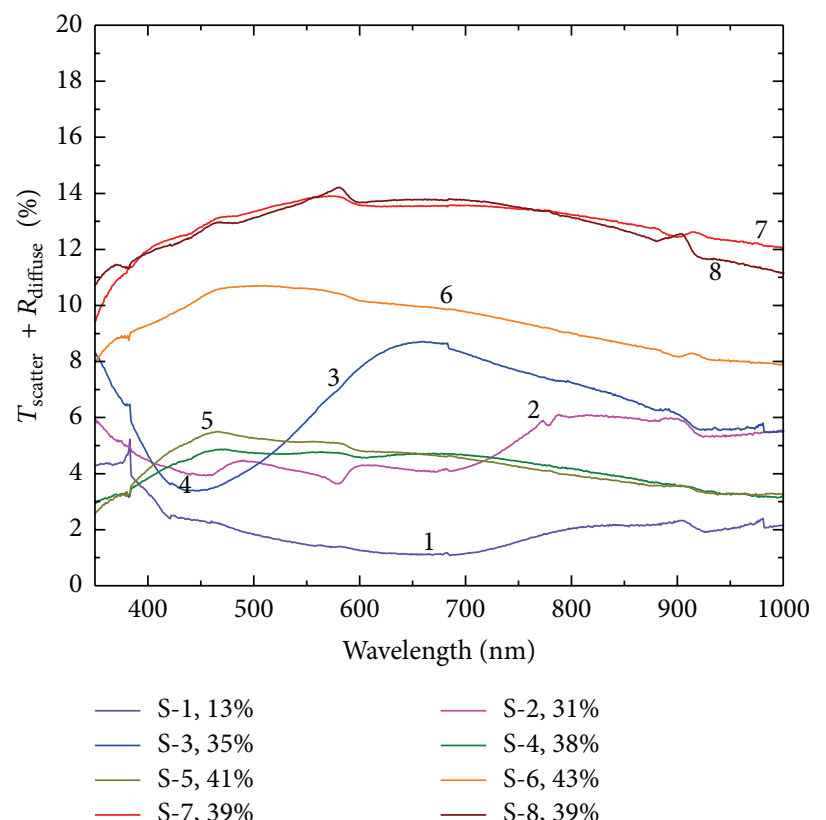

(c)

FIgURE 5: (a) Scattered transmittance, (b) diffuse reflectance, and (c) total scattering (sum of (a) and (b)).

due to the dipole resonance decreases. As the nanoparticle size increases, the absorption comes down and the scattering increases. As in this range, the areal coverage is constant, and an increase in the nanoparticle size results in increased scattering and decreased absorption.

Based on the relationship between thickness and the properties of the nanoparticle films observed so far, silver films of thickness of $\sim 18 \mathrm{~nm}$ were deposited (but this time without the introduction of argon, i.e., at $\sim 5 \times 10^{-6}$ Torr) and were subjected to annealing at $200^{\circ} \mathrm{C}$ for 1 hour. It is seen that the absorptance comes down to $\sim 20 \%$ and the scattering is over $40 \%$ over a broad wavelength range. Thus silver films with thicknesses in this range will be ideally suitable for solar cell applications. The corresponding optical properties are given in Figure 7(a). It is seen that the absorption can be further brought down by annealing the samples in situ without breaking the vacuum. As seen from Figure 7(b), the in situ annealed samples show a lower absorption and also have visible milky appearance.

These films need to be incorporated into thin films solar cell structures to study their effect on the performance of solar cells. Although the overall absorption of silver nanoparticles under this condition is considerably lowered, the lower wavelength absorption might still lead to the lowering of the overall cell performance if the nanoparticles are placed in front of the cell. Hence these nanoparticles can also be incorporated into the backside of thin film solar cell structures. 


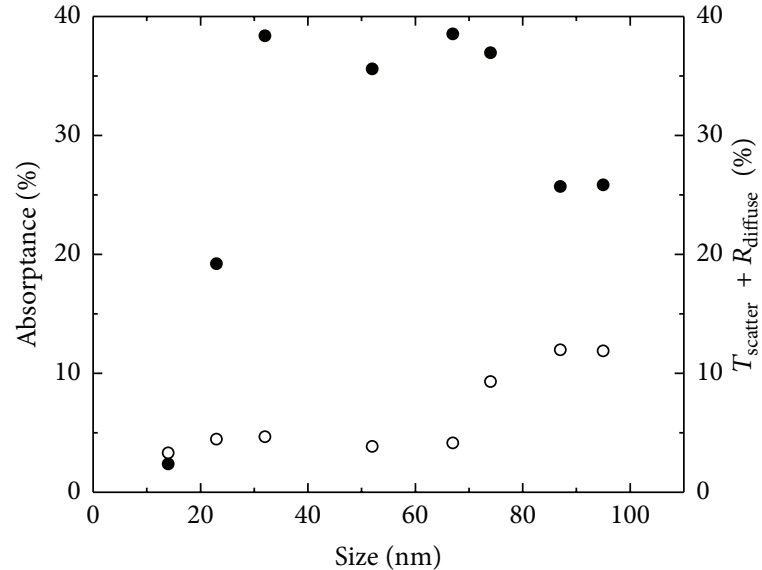

(a) $\lambda=400 \mathrm{~nm}$

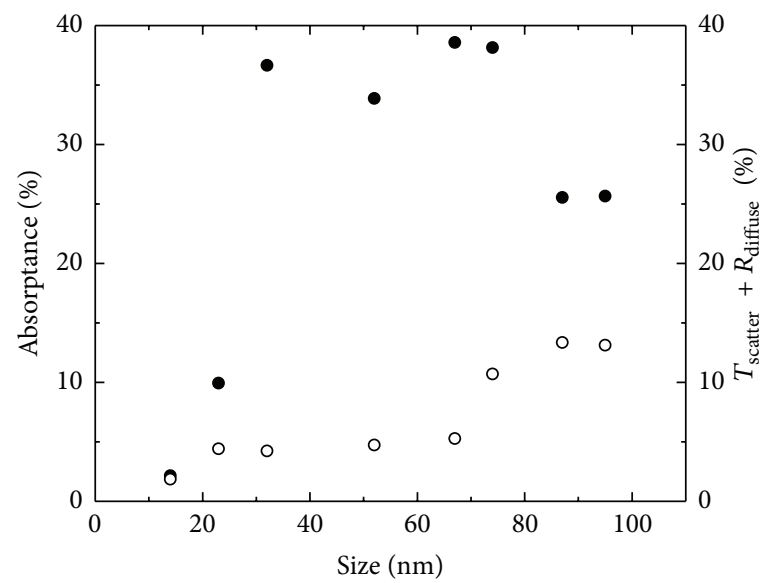

(b) $\lambda=500 \mathrm{~nm}$

FIGURE 6: Absorptance (filled circle) and total scattering (open circle) as a function of effective diameter at $\lambda=400 \mathrm{~nm}$ (a) and (b) $500 \mathrm{~nm}$.

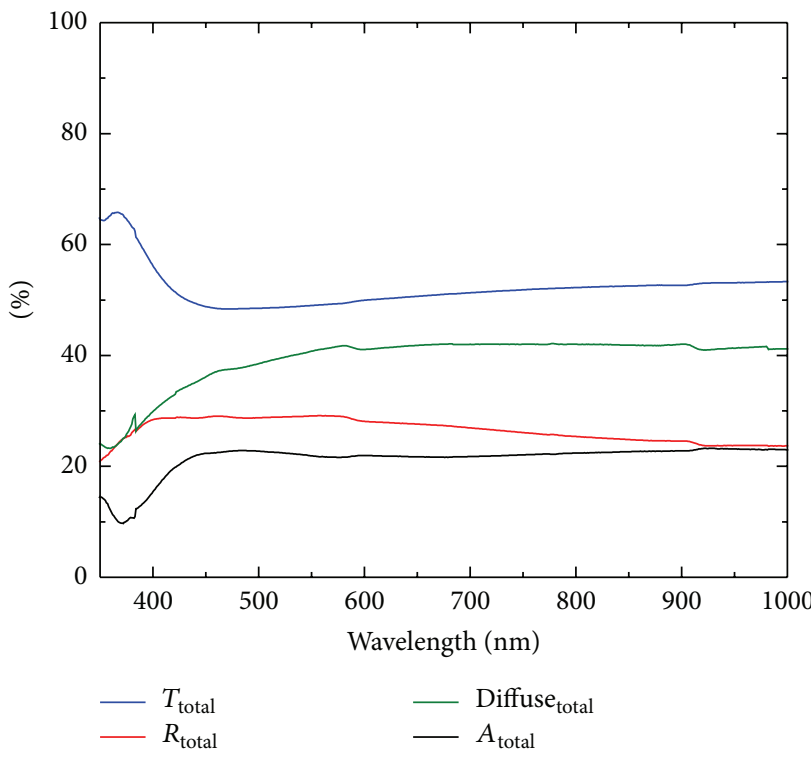

(a) Annealing After 1 day

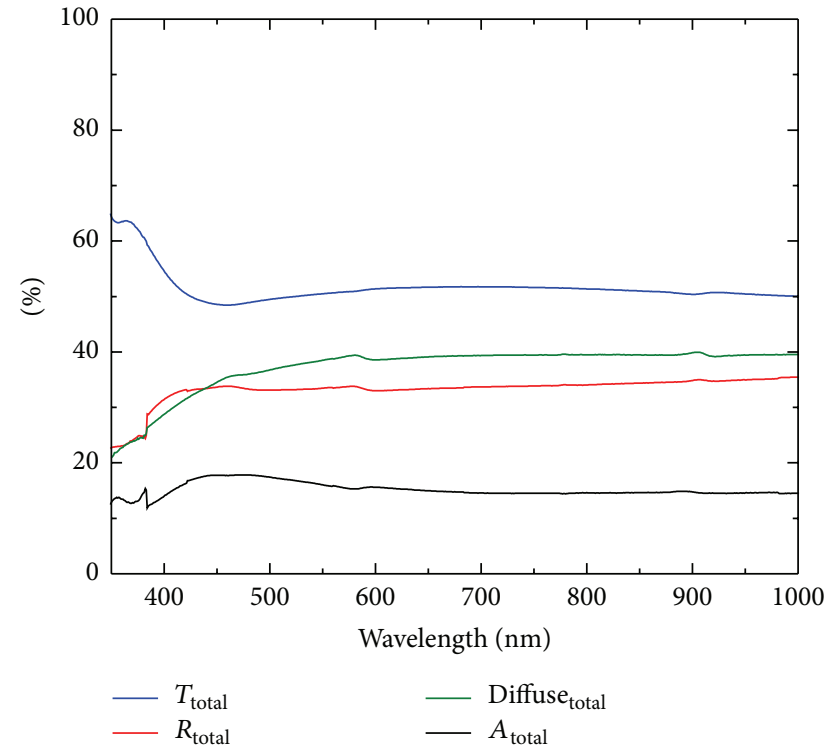

(b) Annealing without breaking vacuum

FiguRE 7: Optical properties of $\sim 18 \mathrm{~nm}$ thick silver films deposited on float glass substrate followed by (a) annealing after 1 day and (b) annealing without breaking vacuum.

\section{Conclusion}

In this work, we have studied the effect of varying the film thickness on the island size and areal coverage of the silver island films. Nanoparticle size increases with increase in film thickness, and the areal coverage increases sharply and saturates at $\sim 40 \%$ for film thicknesses of $\sim 6 \mathrm{~nm}$. The plasmonic effects displayed by the nanoparticles can be clearly observed with the properties being dominated by dipole resonances at lower film thicknesses, which gradually red-shifts as the thickness is increased. Further increase in film thickness results in the appearance of quadrupole modes in addition to the dipole modes. At higher thicknesses, a broad peak encompassing a wide range of wavelengths is observed. This also results in scattering being produced over the entire measured wavelength range of $350 \mathrm{~nm}$ to $1000 \mathrm{~nm}$, but the absorption in the particles is still dominant over the scattering. Increasing the film thickness to $\sim 18 \mathrm{~nm}$ results in further increase in scattering and reduced absorption which should be ideally suited for application in solar cell structures.

Another parameter space that could be of interest is before the islands begin to coalesce. For these films, the optical properties show an increase in scattering at longer wavelengths away from the resonance, where the absorption 
is also lesser. These particles could also be suitable for application in solar cell structures by placing them at the backside of the solar cell.

\section{Acknowledgments}

The authors would like to thank Mr. Amit Gupta for the SEM operation and the creators of the Image J software for making the image processing a lot easier. M. G. Sreenivasan would like to thank the Department of Science and Technology, Government of India, for the support under the project SR/FTP/PS-18/2009 for the work carried out at IIT Delhi.

\section{References}

[1] H. A. Atwater and A. Polman, "Plasmonics for improved photovoltaic devices," Nature Materials, vol. 9, no. 3, pp. 205213, 2010.

[2] A. Polman and H. A. Atwater, "Photonic design principles for ultrahigh-efficiency photovoltaics," Nature Materials, vol. 11, no. 3, pp. 174-177, 2012.

[3] O. Stenzel, A. Stendal, K. Voigtsberger, and C. von Borczyskowski, "Enhancement of the photovoltaic conversion efficiency of copper phthalocyanine thin film devices by incorporation of metal clusters," Solar Energy Materials and Solar Cells, vol. 37, no. 3-4, pp. 337-348, 1995.

[4] H. R. Stuart and D. G. Hall, "Absorption enhancement in silicon-on-insulator waveguides using metal island films," Applied Physics Letters, vol. 69, no. 16, pp. 2327-2329, 1996.

[5] H. R. Stuart and D. G. Hall, "Island size effects in nanoparticleenhanced photodetectors," Applied Physics Letters, vol. 73, no. 26, pp. 3815-3817, 1998.

[6] D. M. Schaadt, B. Feng, and E. T. Yu, "Enhanced semiconductor optical absorption via surface plasmon excitation in metal nanoparticles," Applied Physics Letters, vol. 86, no. 6, Article ID 063106, 3 pages, 2005.

[7] D. Derkacs, S. H. Lim, P. Matheu, W. Mar, and E. T. Yu, "Improved performance of amorphous silicon solar cells via scattering from surface plasmon polaritons in nearby metallic nanoparticles," Applied Physics Letters, vol. 89, no. 9, Article ID 093103, 3 pages, 2006.

[8] S. Pillai, K. R. Catchpole, T. Trupke, and M. A. Green, "Surface plasmon enhanced silicon solar cells," Journal of Applied Physics, vol. 101, no. 9, Article ID 093105, 8 pages, 2007.

[9] K. R. Catchpole and A. Polman, "Design principles for particle plasmon enhanced solar cells," Applied Physics Letters, vol. 93, no. 19, Article ID 191113, 3 pages, 2008.

[10] C. Hägglund, M. Zäch, and B. Kasemo, "Enhanced charge carrier generation in dye sensitized solar cells by nanoparticle plasmons," Applied Physics Letters, vol. 92, no. 1, Article ID 013113, 3 pages, 2008.

[11] S. S. Kim, S. I. Na, J. Jo, D. Y. Kim, and Y. C. Nah, "Plasmon enhanced performance of organic solar cells using electrodeposited Ag nanoparticles," Applied Physics Letters, vol. 93, no. 7, Article ID 073307, 3 pages, 2008.

[12] K. Nakayama, K. Tanabe, and H. A. Atwater, "Plasmonic nanoparticle enhanced light absorption in GaAs solar cells," Applied Physics Letters, vol. 93, no. 12, Article ID 121904, 3 pages, 2008.
[13] S. Varlamov, Z. Ouyang, X. Zhao, and D. S. Jung, "Surface plasmon enhanced light-trapping in polycrystalline silicon thinfilm solar," in Proceedings of the Photonics Global Conference (PGC '10), pp. 1-6, December 2010.

[14] R. Najjar, E. Quesnel, N. Baclet et al., "Improvement of energy conversion efficiency of amorphous silicon thin-film solar cells through plasmon effect," in Proceedings of the MIDEM 2009 Conference, pp. 281-286, 2009.

[15] C. Eminian, F. J. Haug, O. Cubero, X. Niquille, and C. Ballif, "Photocurrent enhancement in thin film amorphous silicon solar cells with silver nanoparticles," Progress in Photovoltaics, vol. 19, no. 3, pp. 260-265, 2011.

[16] E. Moulin, J. Sukmanowski, M. Schulte, A. Gordijn, F. X. Royer, and H. Stiebig, "Thin-film silicon solar cells with integrated silver nanoparticles," Thin Solid Films, vol. 516, no. 20, pp. 68136817, 2008.

[17] X. Chen, B. Jia, J. K. Saha et al., "Broadband enhancement in thin-film amorphous silicon solar cells enabled by nucleated silver nanoparticles," Nano Letters, vol. 12, no. 5, pp. 2187-2192, 2012.

[18] ImageJ, http://rsbweb.nih.gov/ij/ .

[19] J. P. Clarkson, Plasmon enhanced absorption in photovoltaic cells [Ph.D. thesis], University of Rochester, 2010.

[20] S. A. Maier, M. L. Brongersma, P. G. Kik, and H. A. Atwater, "Observation of near-field coupling in metal nanoparticle chains using far-field polarization spectroscopy," Physical Review B, vol. 65, no. 19, Article ID 193408, 4 pages, 2002.

[21] K. H. Su, Q. H. Wei, X. Zhang, J. J. Mock, D. R. Smith, and S. Schultz, "Interparticle coupling effects on plasmon resonances of nanogold particles," Nano Letters, vol. 3, no. 8, pp. 1087-1090, 2003.

[22] P. K. Jain, W. Huang, and M. A. El-Sayed, "On the universal scaling behavior of the distance decay of plasmon coupling in metal nanoparticle pairs: a plasmon ruler equation," Nano Letters, vol. 7, no. 7, pp. 2080-2088, 2007.

[23] S. Berciaud, L. Cognet, P. Tamarat, and B. Lounis, “Observation of intrinsic size effects in the optical response of individual gold nanoparticles," Nano Letters, vol. 5, no. 3, pp. 515-518, 2005.

[24] K. R. Catchpole and A. Polman, "Plasmonic solar cells," Optics Express, vol. 16, no. 26, pp. 21793-21800, 2008.

[25] Y. A. Akimov, W. S. Koh, and K. Ostrikov, "Enhancement of optical absorption in thin-film solar cells through the excitation of higher-order nanoparticle plasmon modes," Optics Express, vol. 17, no. 12, pp. 10195-10205, 2009. 

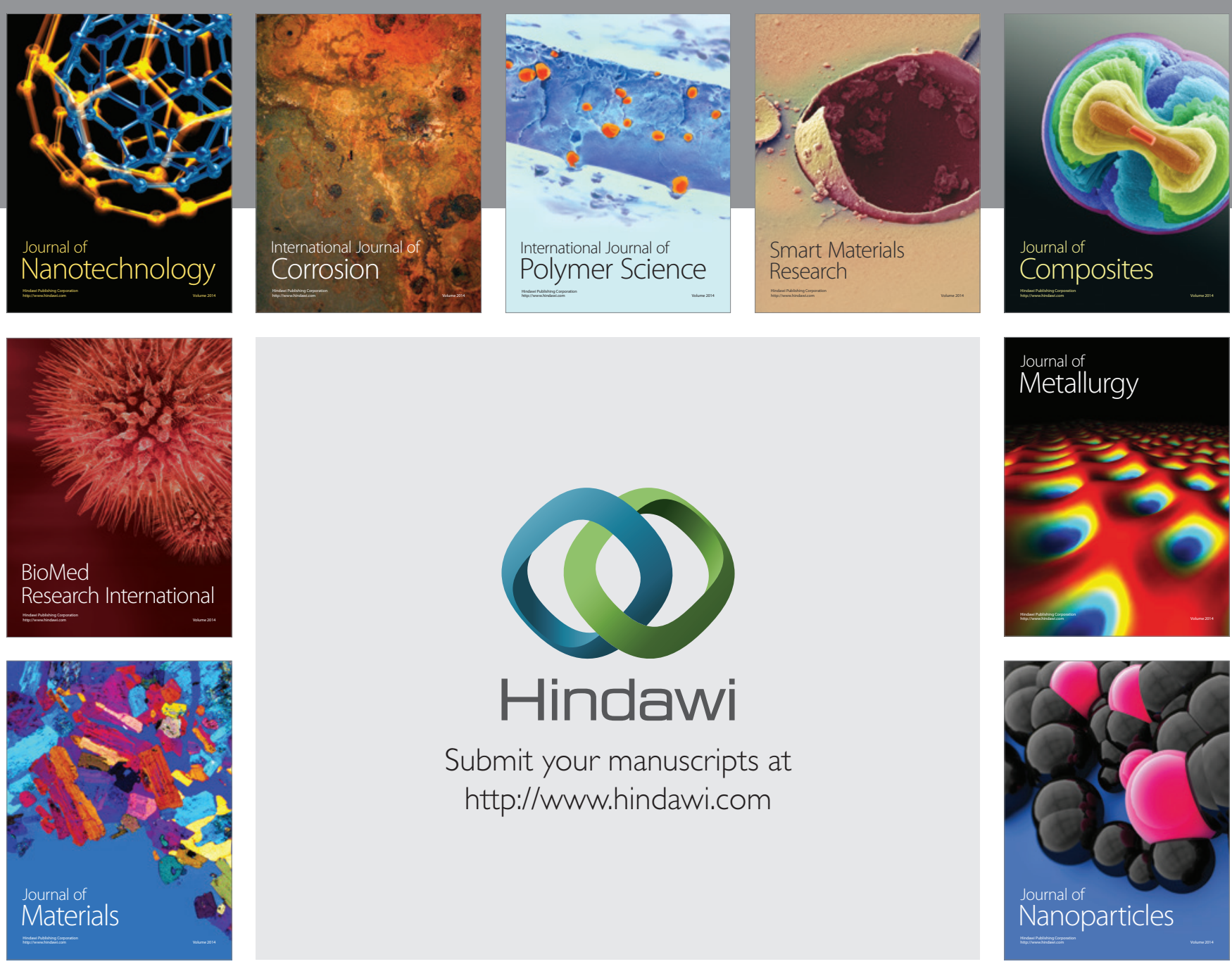

Submit your manuscripts at http://www.hindawi.com
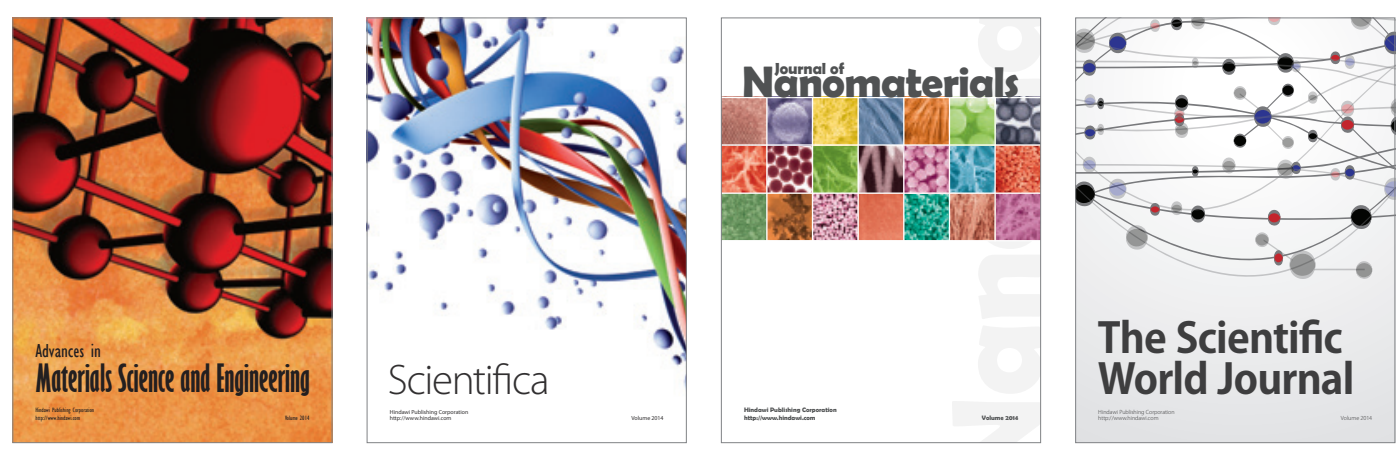

\section{The Scientific World Journal}
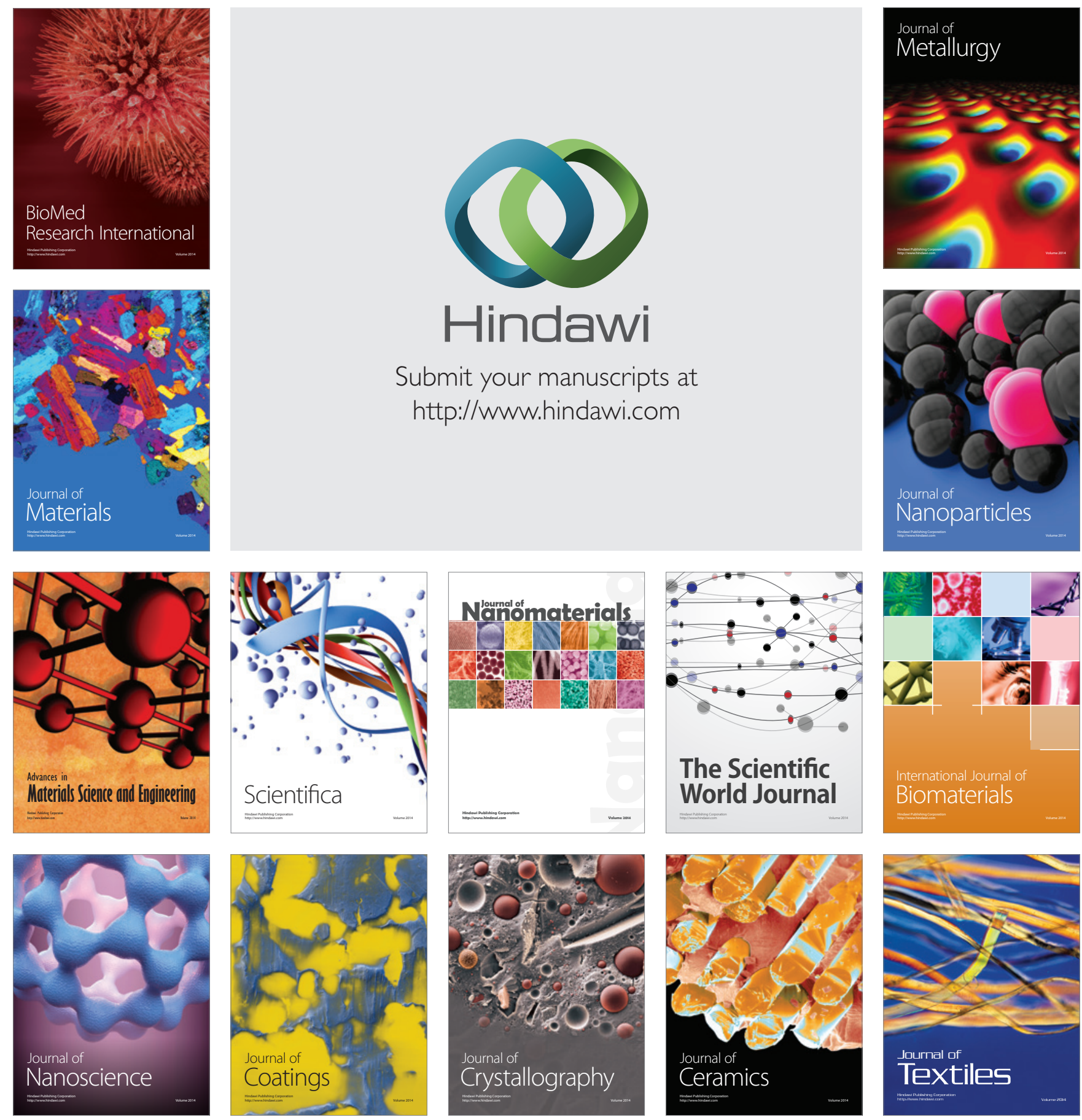